\title{
AJVI.
}

\section{Challenges of Integrated Navigation}

\section{Dr Paul D Groves}

University College London

(p.groves@ucl.ac.uk)

Panel: Multisensor Fusion for

Advanced Navigation

ION GNSS+ 2018

28 September 2018

\section{UCL ENGINEERING}

Change the world

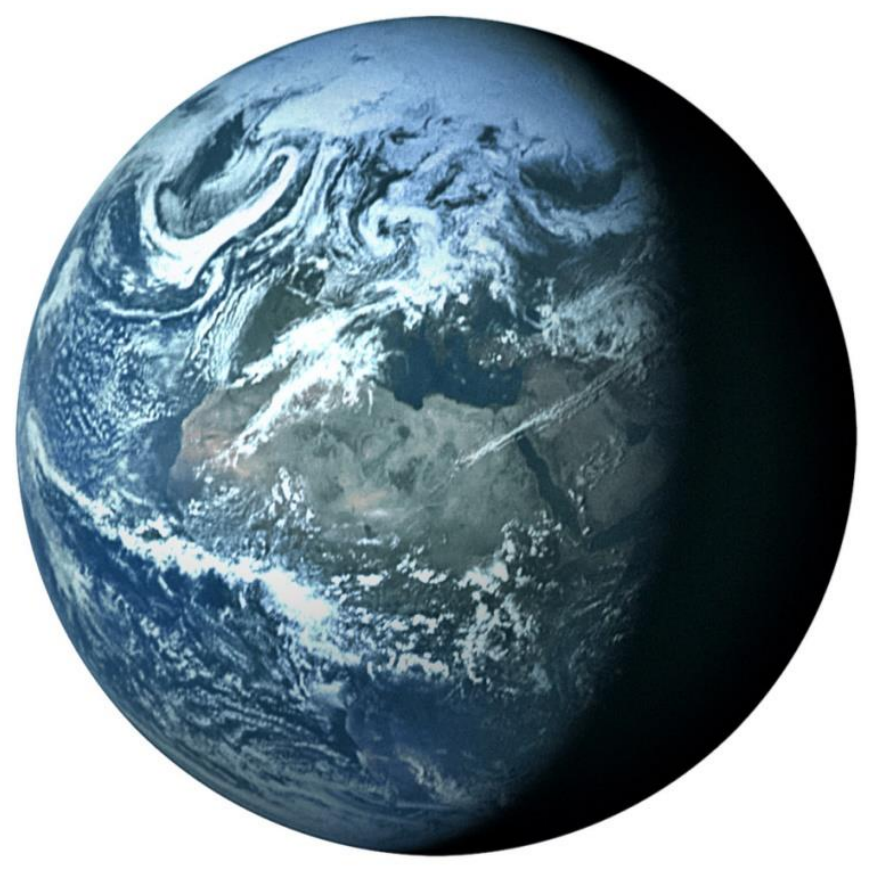




\section{$\triangle V \mid C L_{1}$}

\section{Latest Navigation Requirements}

- Meter accuracy

- Autonomous vehicles

- Drones

- Visually impaired pedestrians

- Seamless/ ubiquitous positioning

- Pedestrians and vehicles

- Indoors and outdoors
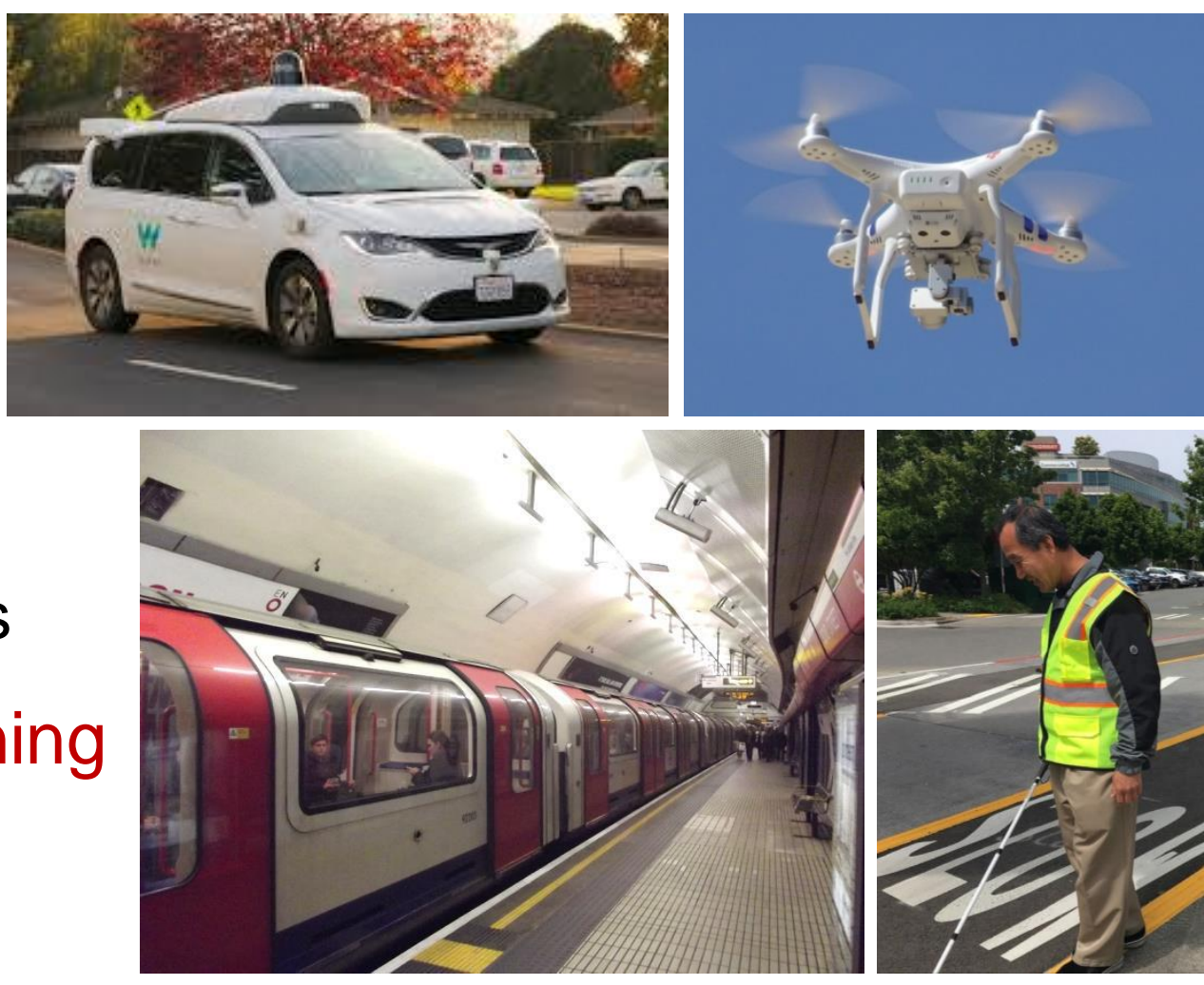

- Resilience

- Environments with limited signal reception

- Jamming, interference and spoofing

- Integrity
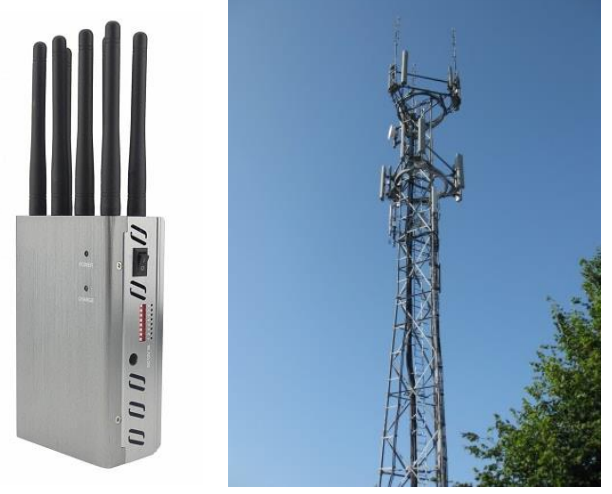


\section{Technology Background}

- Smaller cheaper sensors

- IMUs in every smartphone

- HD video cameras in every phone

- Magnetometers, barometers, ambient light

- More processing capacity

- But for how long?

- More data available

- 3D mapping

- Streetview

- Signal databases
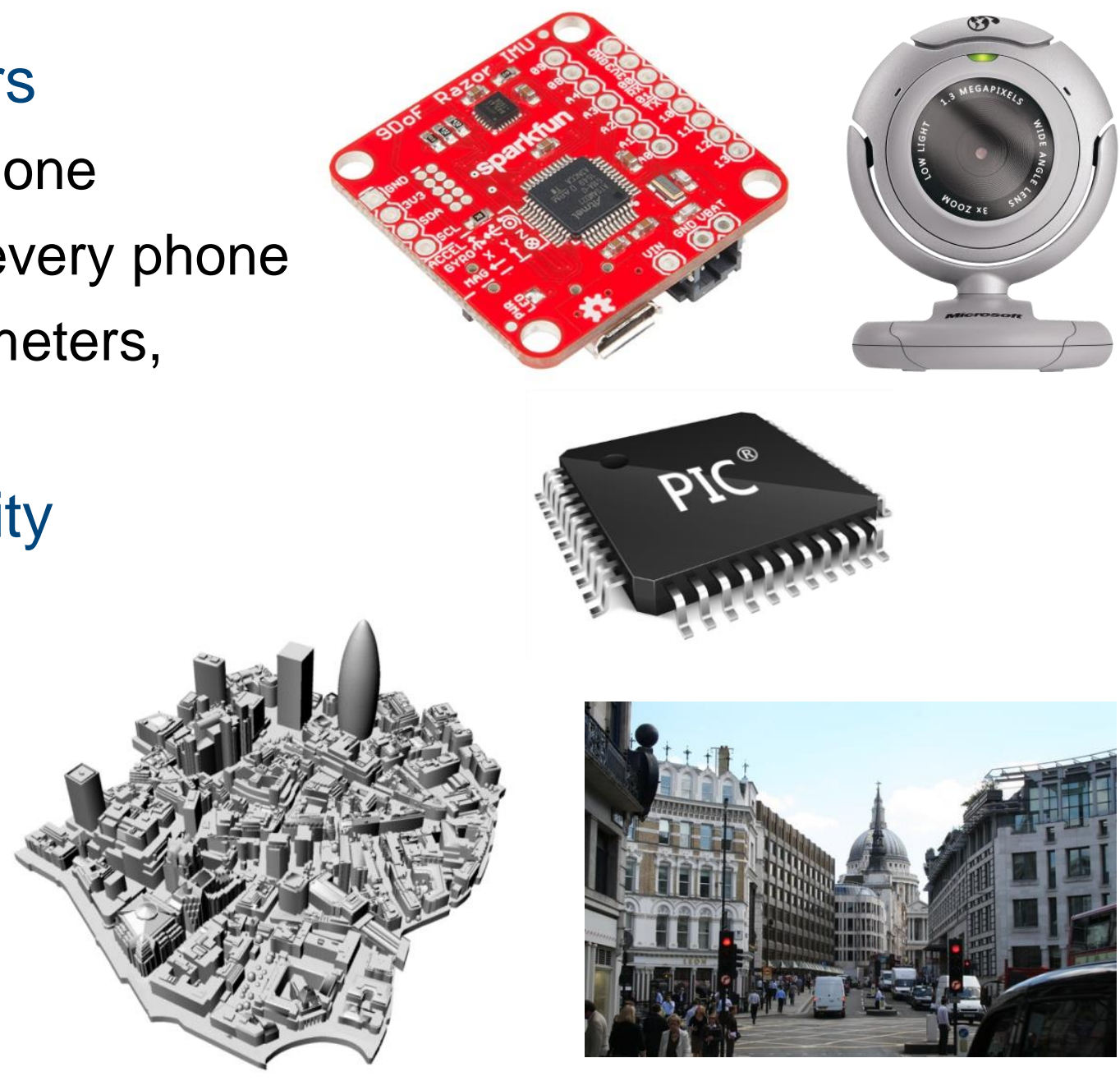

0
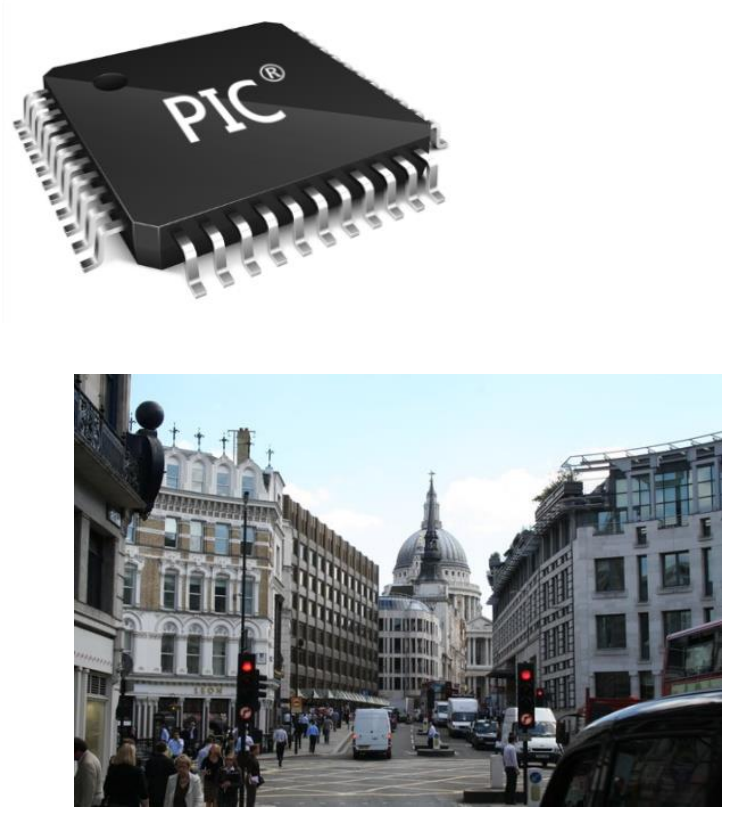


\section{$\triangle I / C L_{1}$}

\section{PNT Technology Developments}

\section{GNSS}

- New signals and multi-frequency receivers

- Shadow matching and 3D mapping aided ranging

- Please come to Session E6 at 3:20 this afternoon (:)

- Extended coherent integration and synthetic aperture beamforming

\section{Other technologies}

- Wi-Fi Round Trip Time (802.11mc)

- Magnetic anomaly matching

- 5G communications

- LEO communication satellites

- Better visual navigation

- More accurate pedestrian dead reckoning
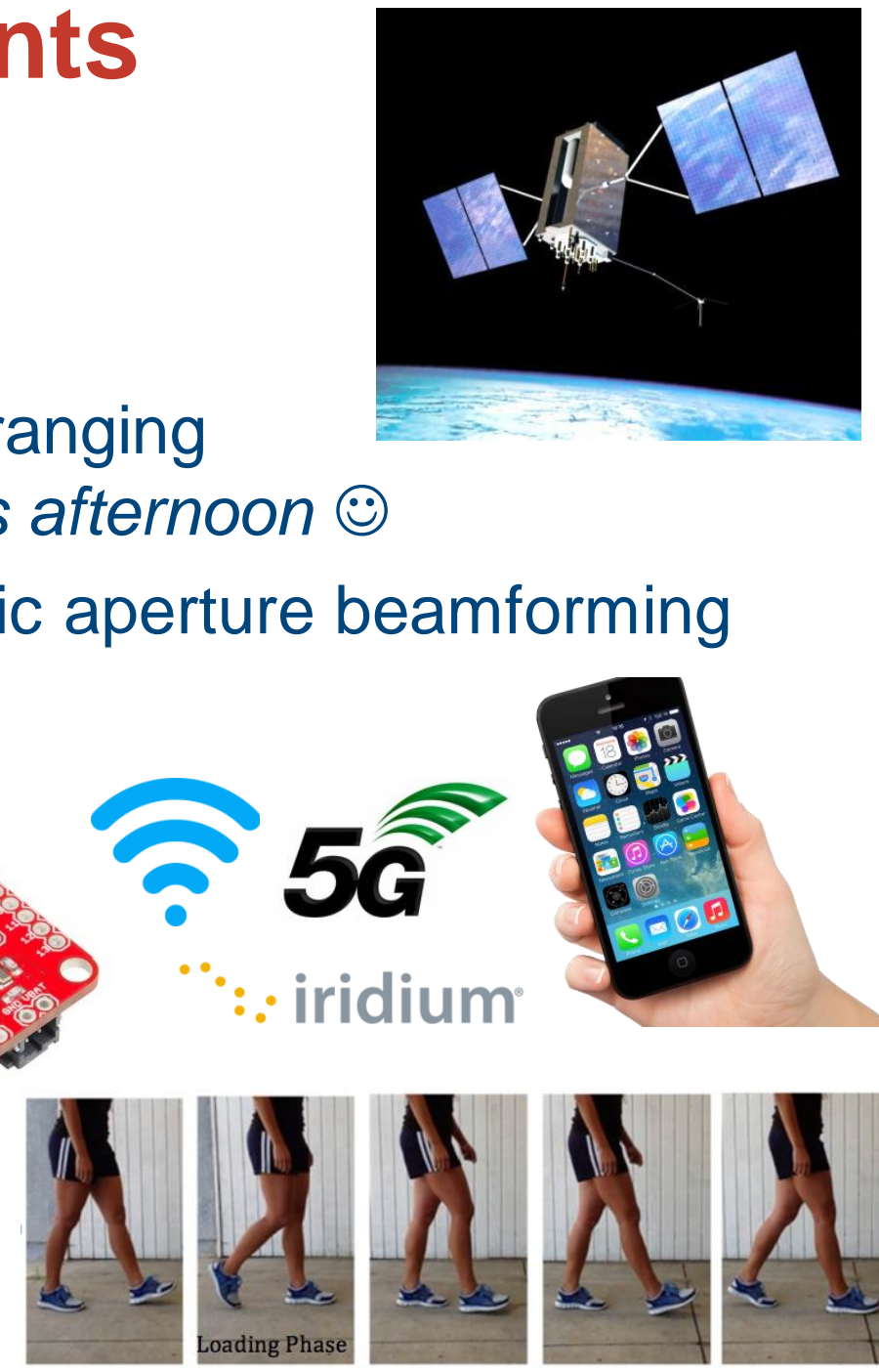

- Better MEMS inertial sensors and quantum sensor technology 
GNSS and Other Radio Signals:

Jamming

Spoofing

Interference


Signals not always available

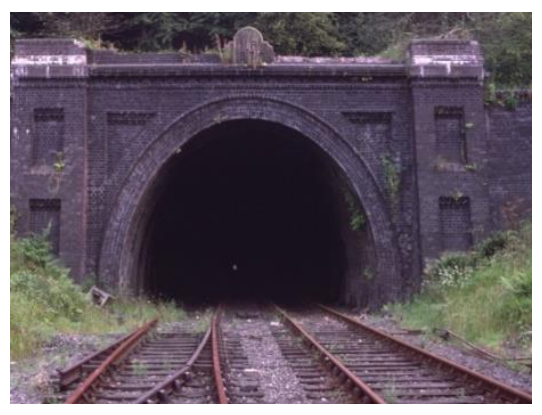

Visual Navigation:

Landmarks are not available everywhere

Dead Reckoning:

Errors grow with time

Things Break:

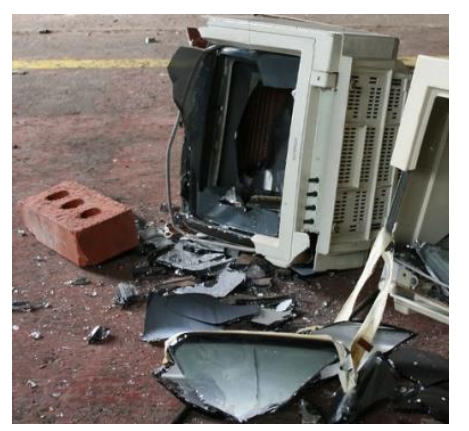




\section{$\triangle \mathrm{J} / \mathrm{CL}$}

\section{The Quantum Sensor Myth}

Performance claims include

"One meter per month" inertial navigation drift

BUT... even with perfect sensors, we have:

1. Initialisation errors

- A $1 \mathrm{~mm}$ height initialisation error will

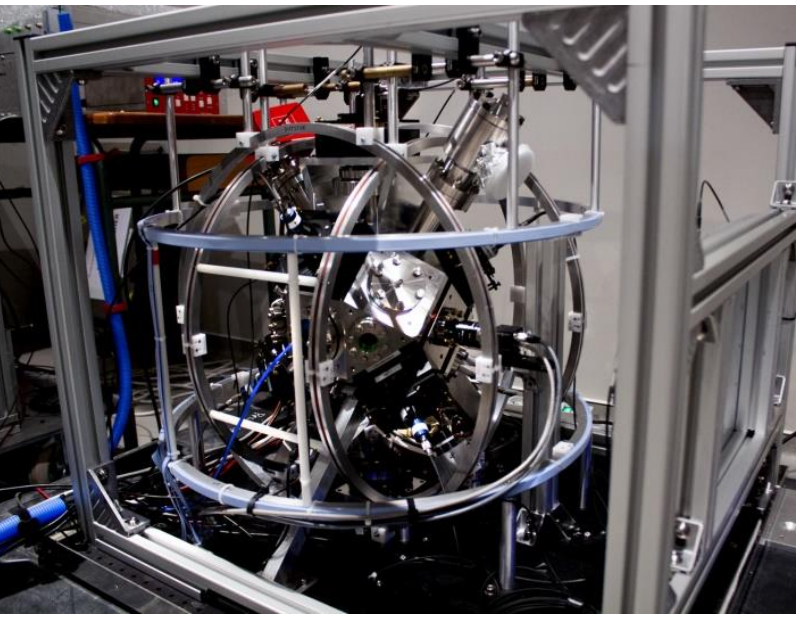
grow to $1 \mathrm{~km}$ after 75 minutes

2. Errors due to mounting misalignment

- Flexure effects not easily calibrated

3. Gravity modelling errors

- EGM2008 accuracy equivalent to $20 \mathrm{~m}$ position error after 1000s

4. Errors due to bandwidth limitation

5. Numerical rounding errors

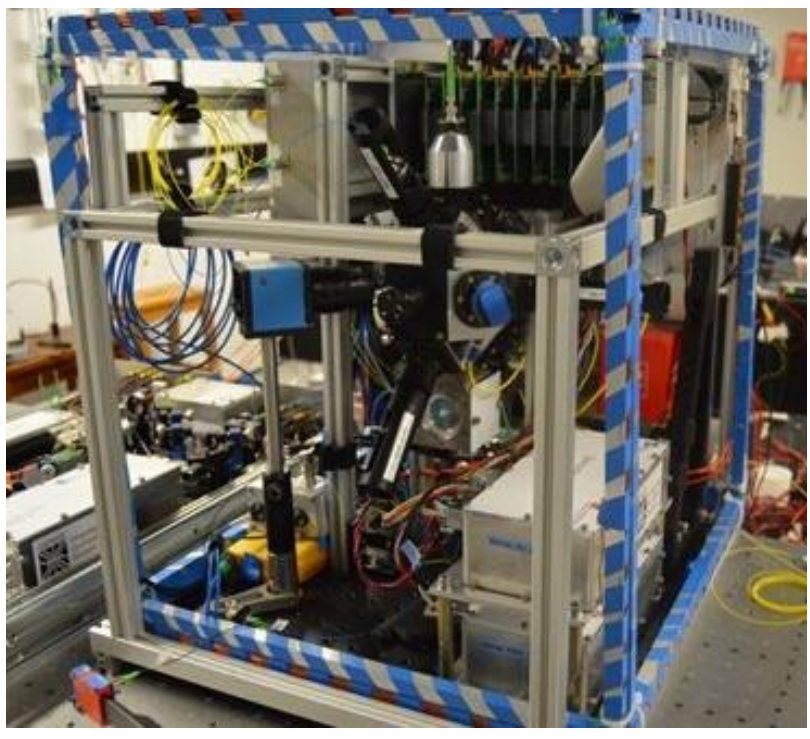

$\therefore$ Error-free inertial navigation is not possible

Top: LP2N Bordeaux and LNE-SYRTE Paris Bottom: University of Birmingham 


\section{We still need Integrated Navigation}

\section{Benefits:}

- Resilience: With enough different technologies, we can always maintain a navigation solution

- Accuracy: More information enables greater accuracy and better sensor error calibration

- Integrity: More information makes faults easier to spot

Increased complexity brings challenges:

- Expertise: Bringing together knowledge of many different technologies

- Upgrades: How to incorporate new technology without a complete redesign

- Integrity: How do we ensure the navigation solution can be trusted

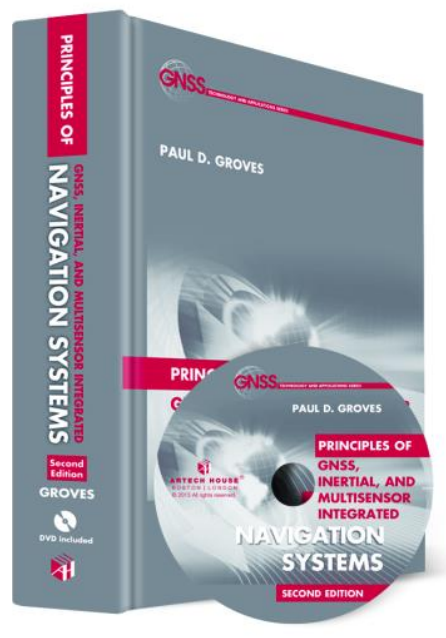




\section{Many Different Navigation Technologies}

$\geq 13$ smartphone

pedestrian

positioning

techniques

Other platforms

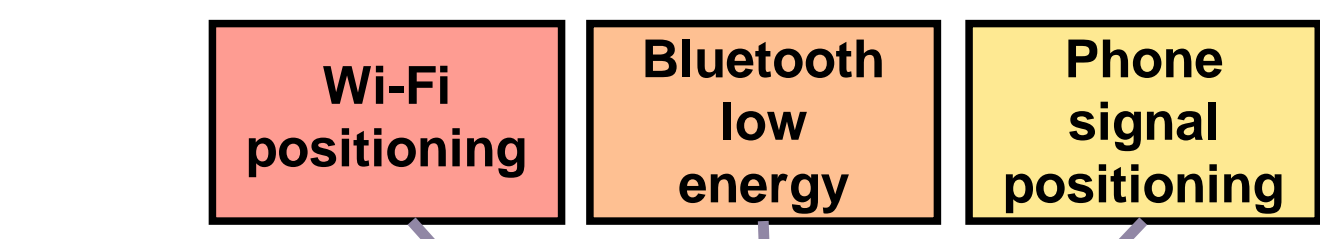

use other

techniques

How do we select the best techniques and combine them efficiently?
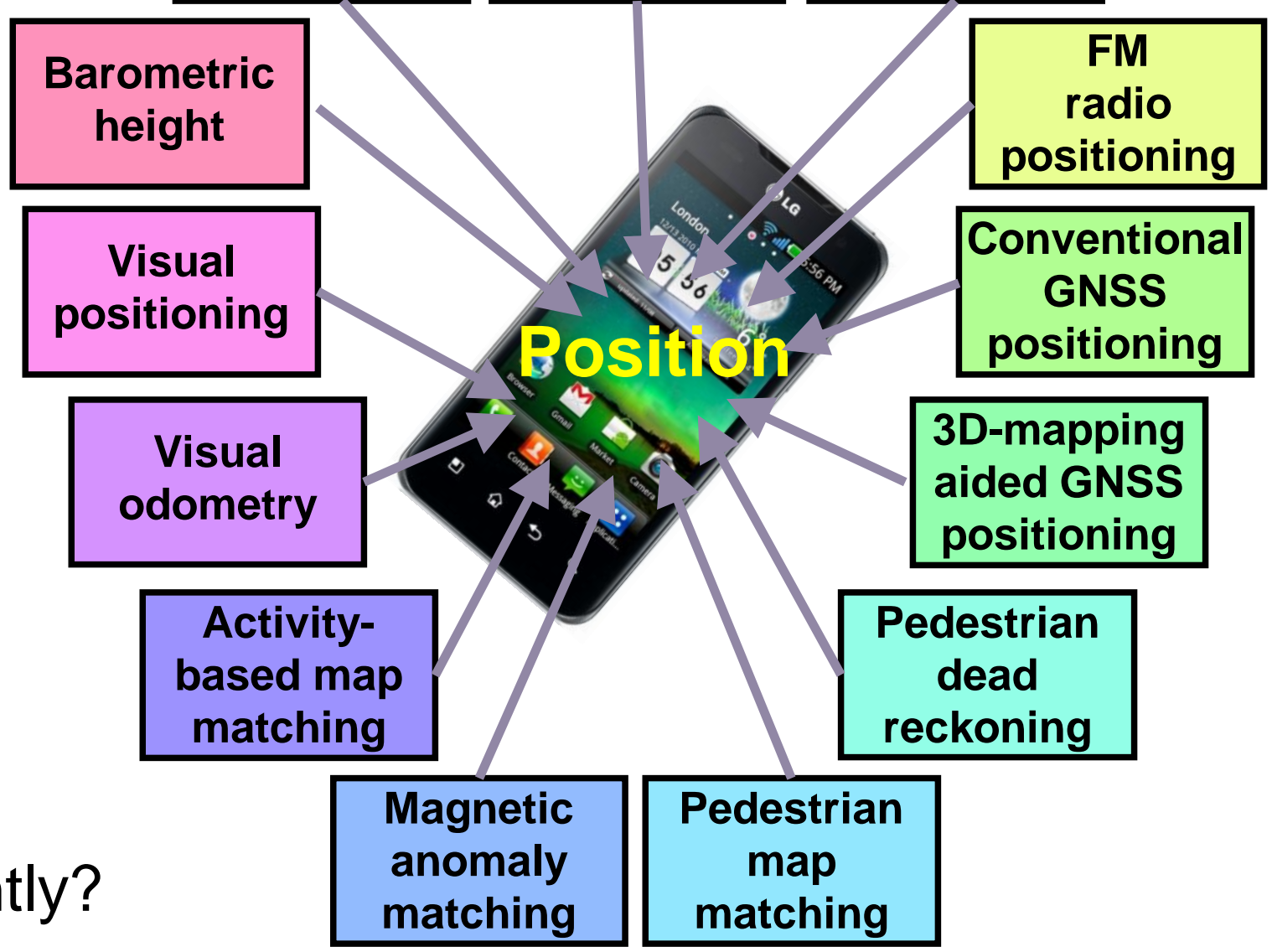


\section{Context is Important}

It determines which navigation technologies work best

\section{Environment}
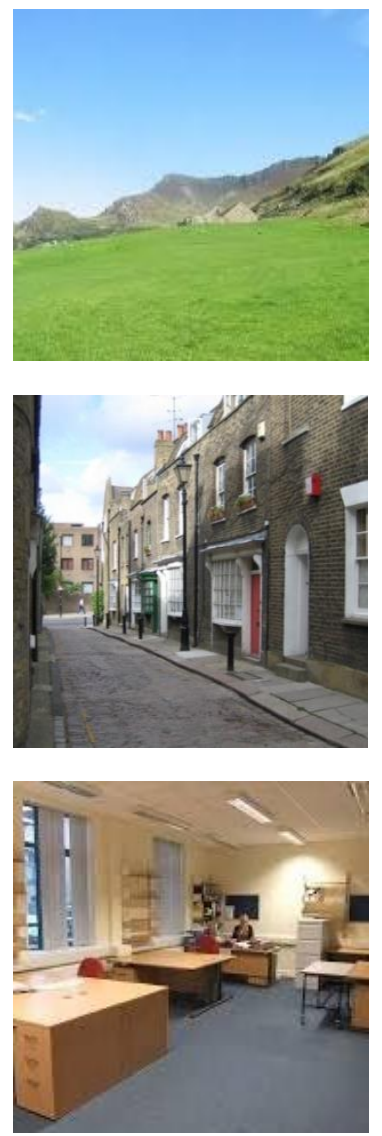

Open: Standard GNSS works well

Urban: Use 3Dmapping aided GNSS

Indoor: Wi-Fi generally best
Behaviour
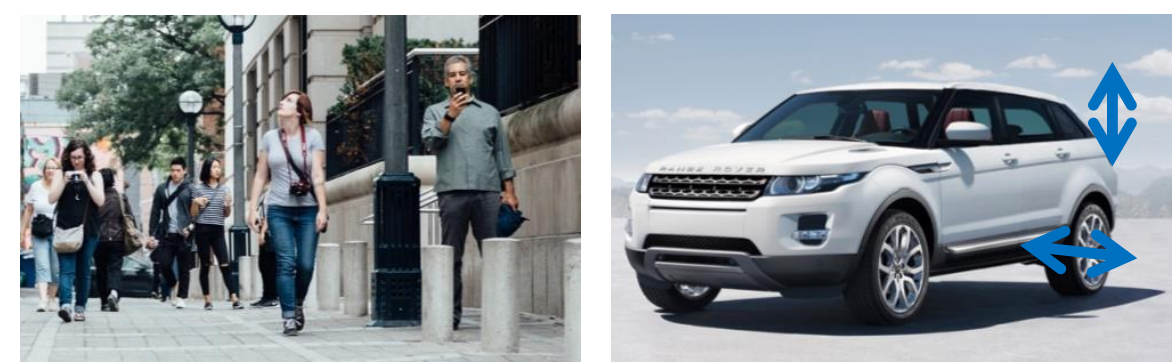

Pedestrians and Vehicles

- Different map matching

- Different motion constraints

- Step detection only works for pedestrians 


\section{Context-Adaptive Navigation}

- Detects the environmental and behavioural context.

- Selects the appropriate navigation techniques

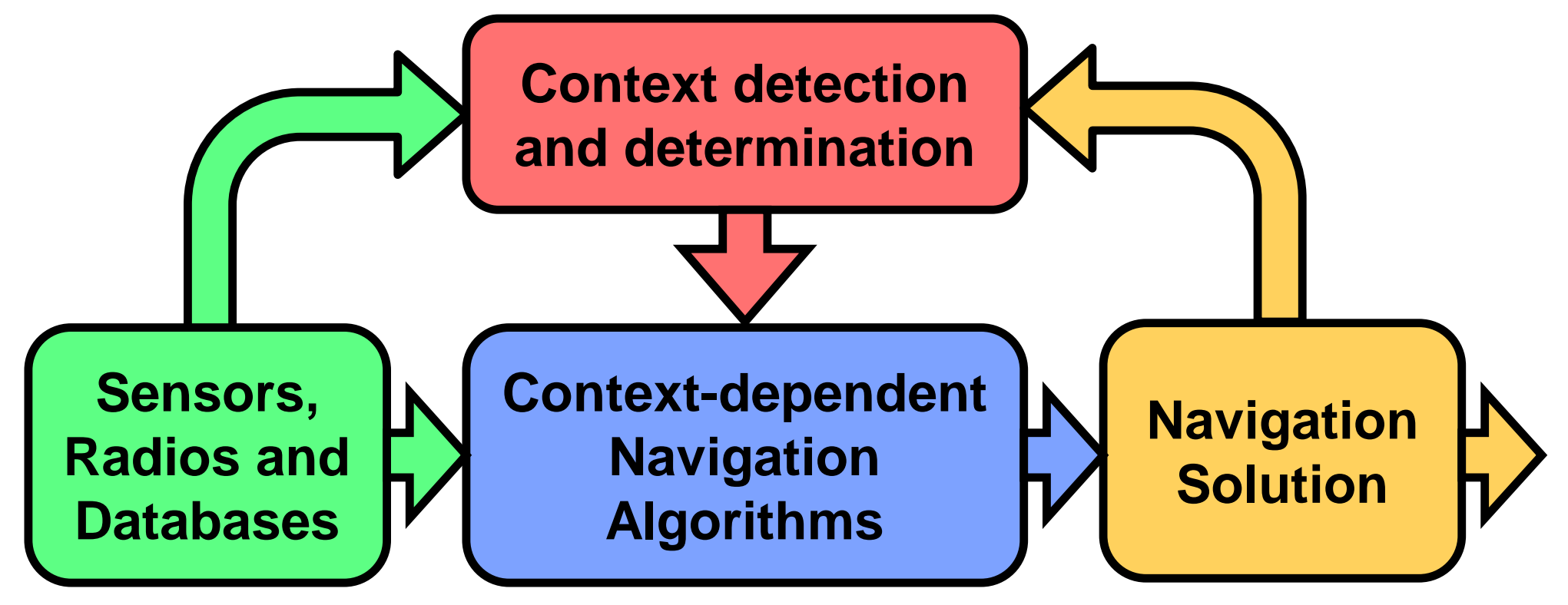

Please come to Han Gao's presentation at 1:50 in Session E6 (:) 


\section{$\triangle I / C L_{1}$}

\section{Conventional Integrated Navigation (1)}

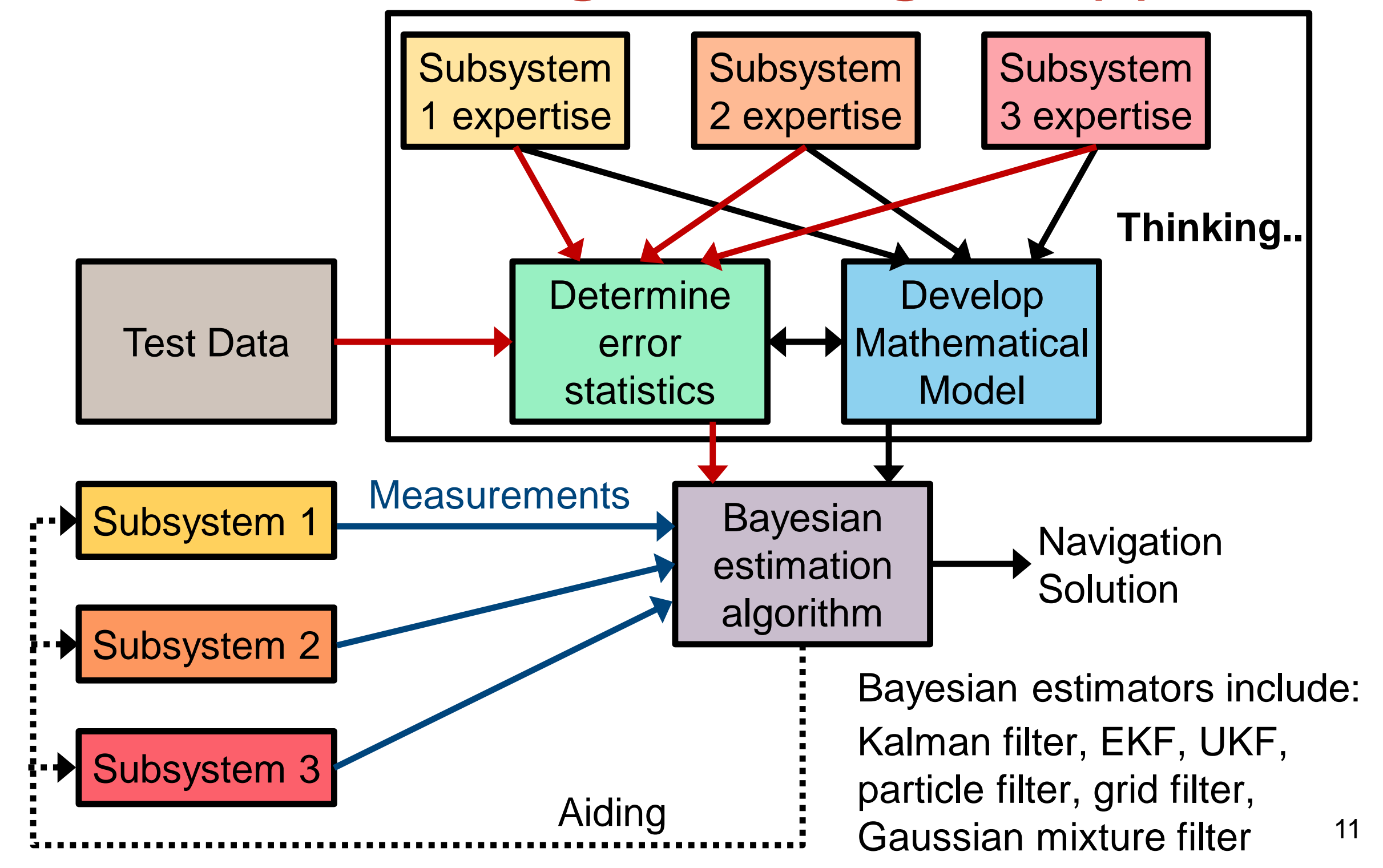




\section{Conventional Integrated Navigation (2)}

\section{Challenges:}

1. How do we bring together all of the subsystem and modelling expertise?

2. How do we avoid a complete redesign when we add or change a subsystem?
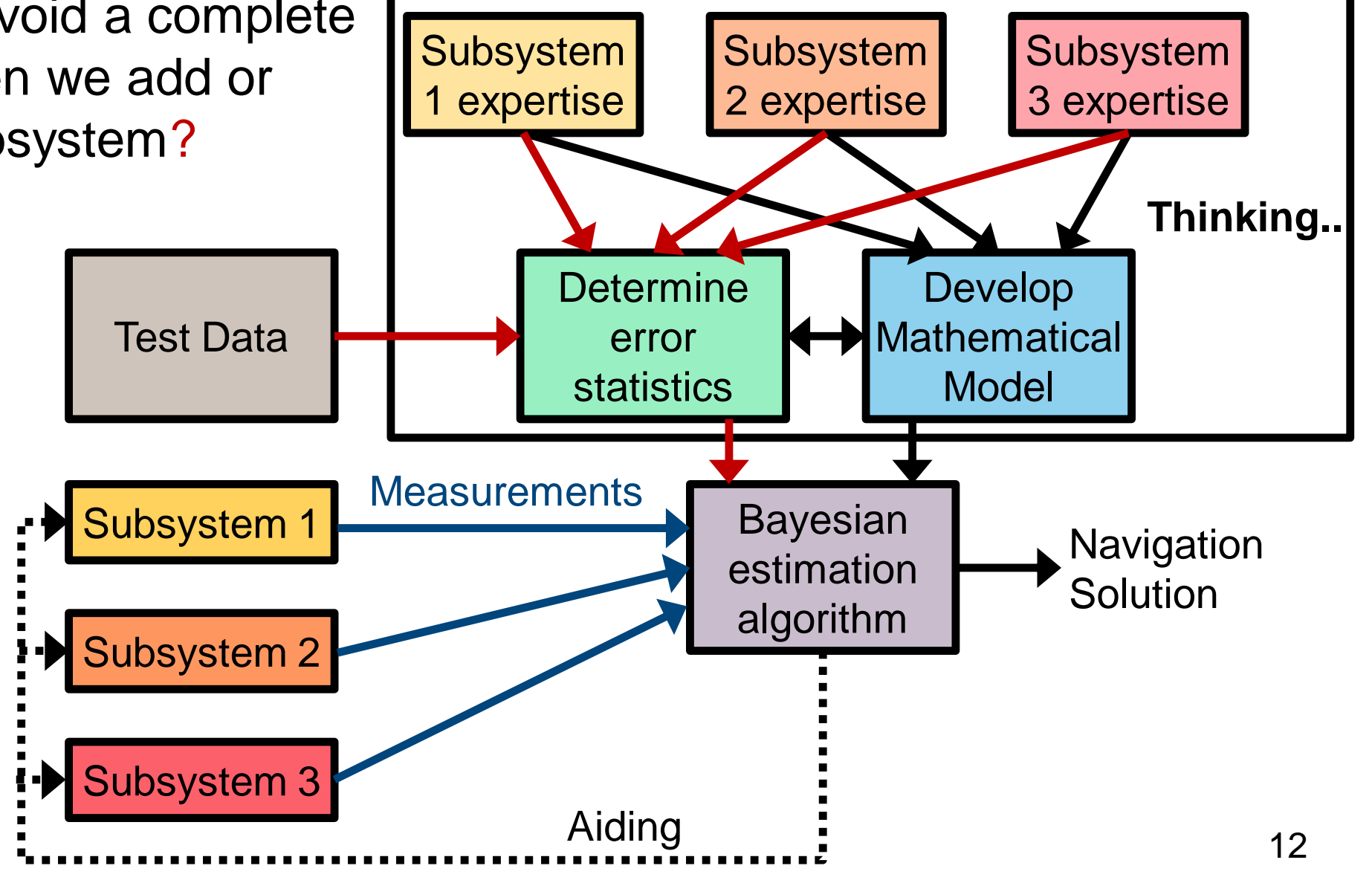


\section{Plug'n'Play Integrated Navigation (1)}
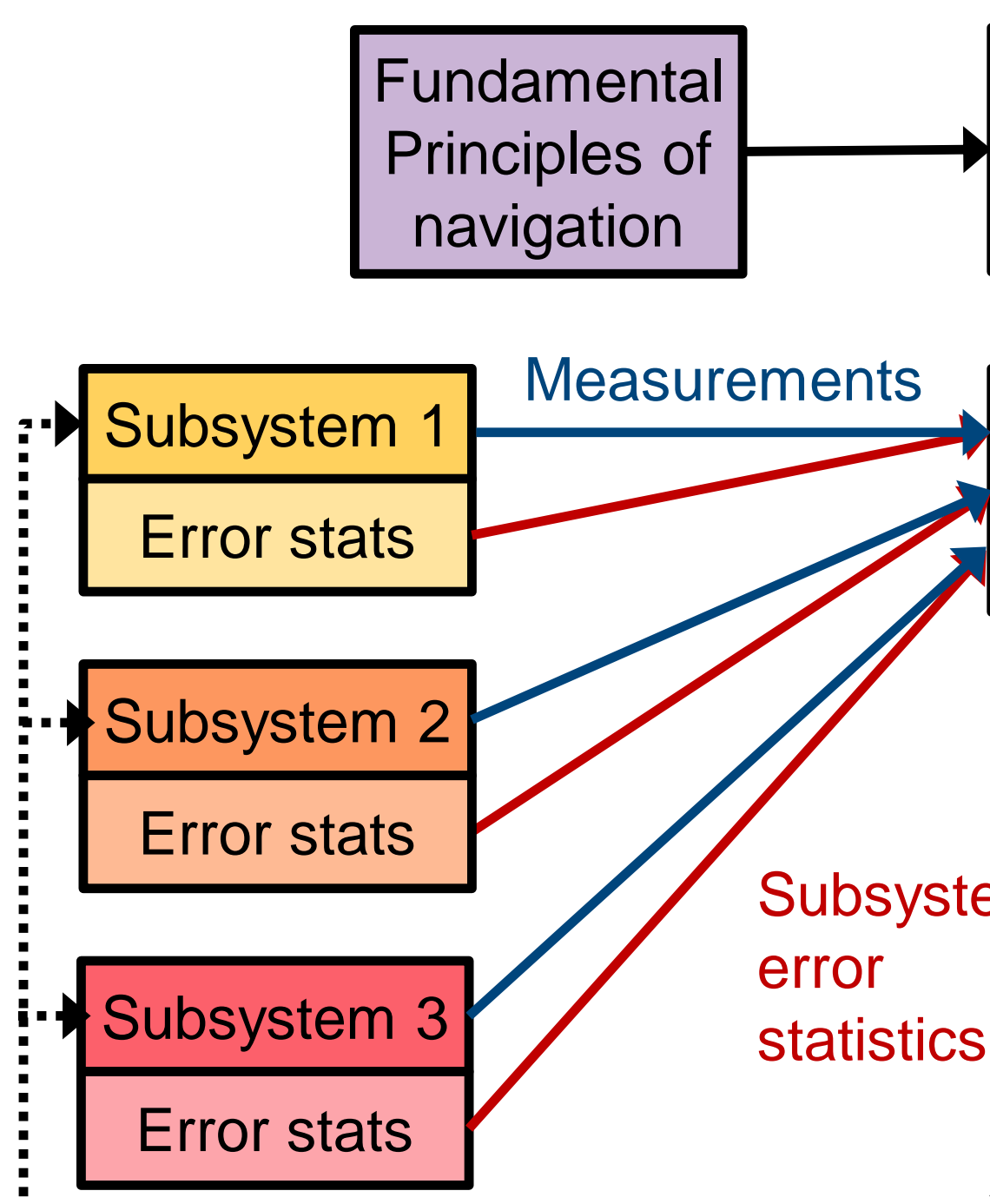

"Universal" Mathematical

Model

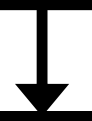

\section{Measurements}

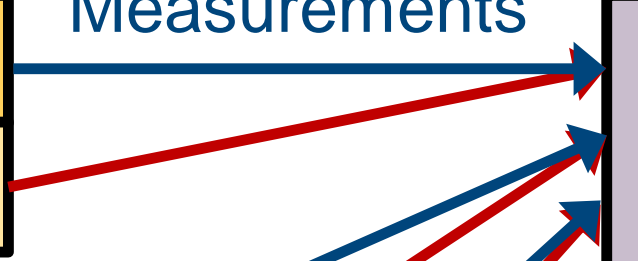

Bayesian estimation algorithm
Expertise and thinking distributed across integration algorithm and subsystems

\section{Estimator inputs standard} measurement types:

- Position and velocity

Subsystem error statistics

- (Pseudo-)Ranges

- Landmark directions

- Position likelihood surface Aiding - Specific force and angular rate... 


\section{Plug'n'Play Integrated Navigation (2)}



Groves, P. D., The Complexity Problem in Future Multisensor Navigation and Positioning Systems: A Modular Solution. Journal of Navigation, 67 (2), 2014, 311-326.

Groves, P. D. et al. The Four Key Challenges of Advanced Multisensor Navigation and Positioning, Proc. IEEE/ION PLANS 2014.
Expertise and thinking distributed across integration algorithm and subsystems

\section{Challenges:}

1. Including every measurement type in the "universal" model

2. Agreeing the interface

3. Ensuring the subsystem manufacturers' error statistics are trustworthy 


\section{Self-Calibrating Plug'n'Play Integration (1)}

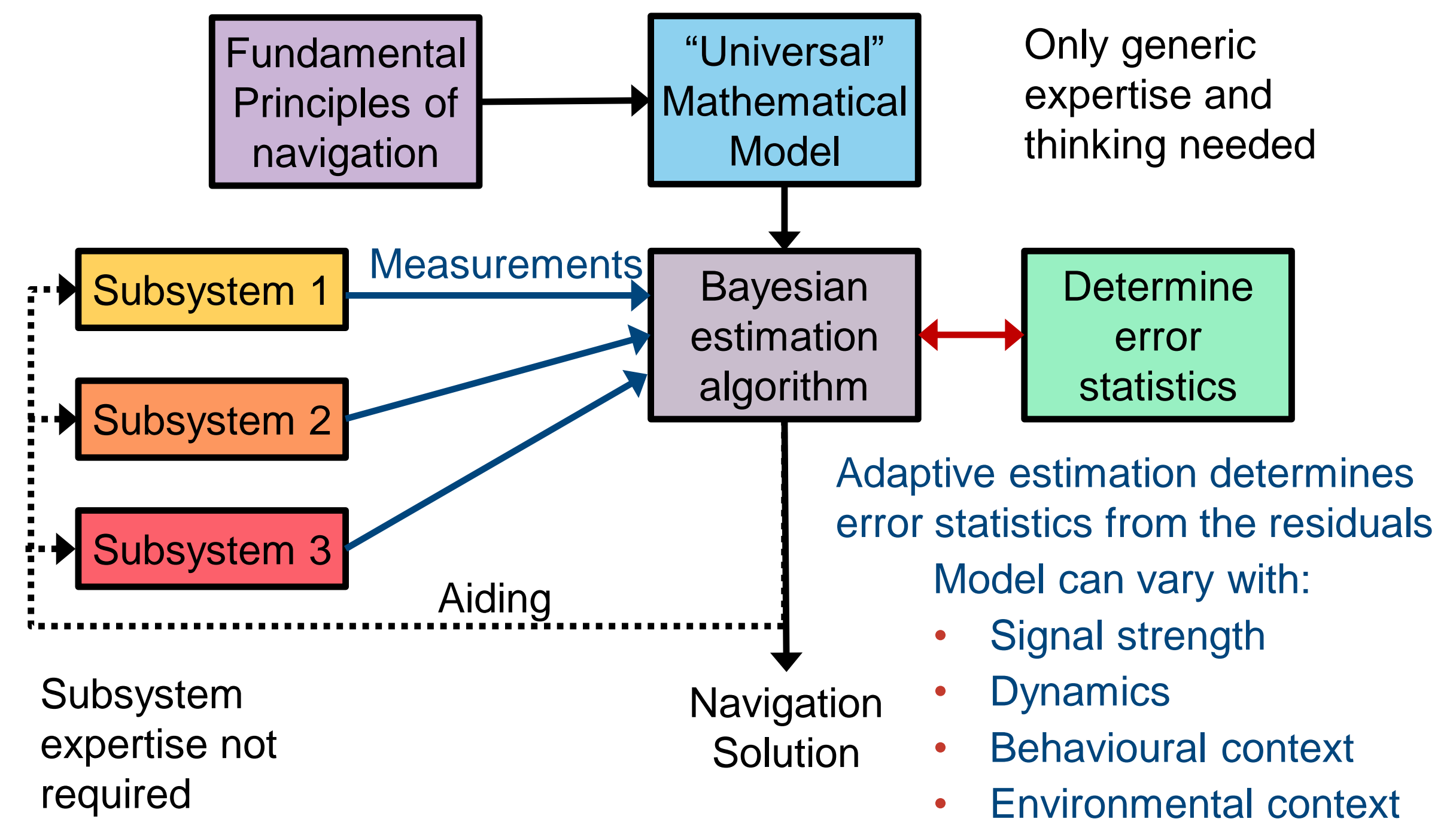




\section{Self-Calibrating Plug'n’Play Integration (2)}

\section{Challenges:}

1. Including every measurement type in the "universal" model

2. Agreeing the interface (though at least it's simpler)

3. Capturing enough data to reliably determine the error statistics

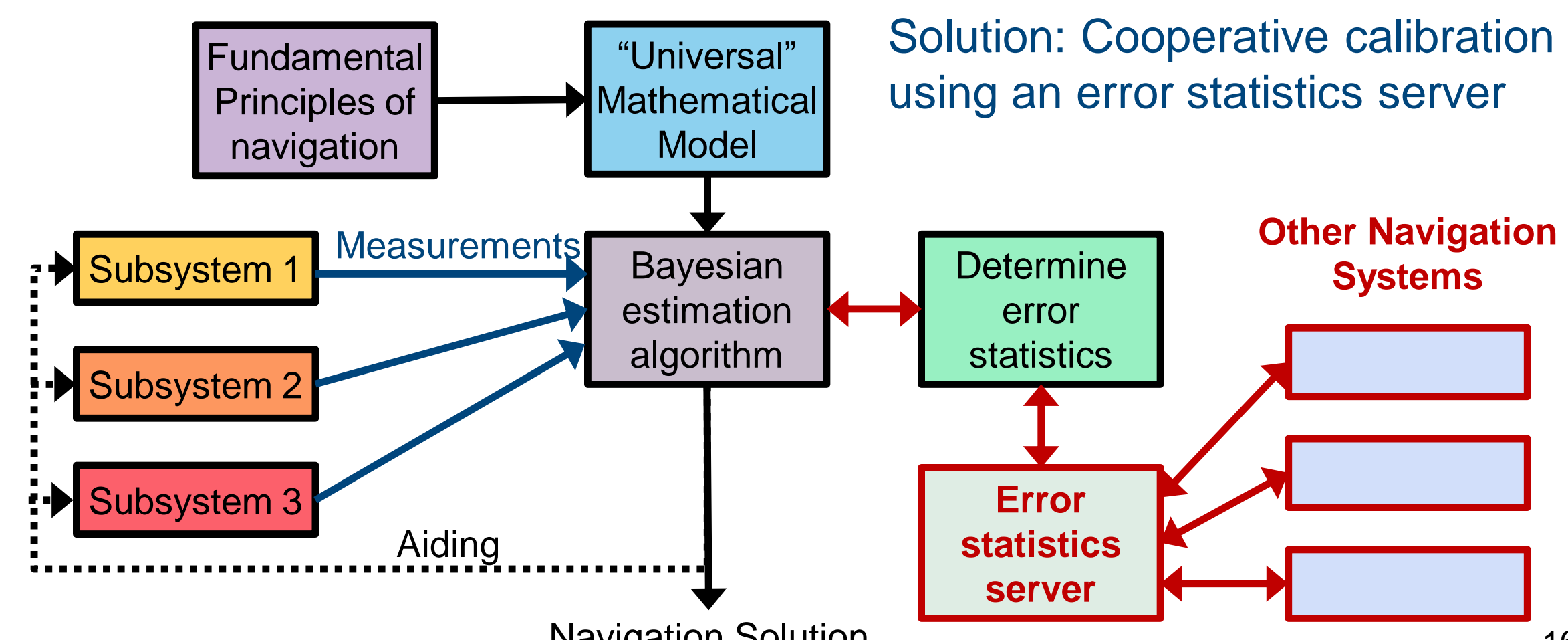




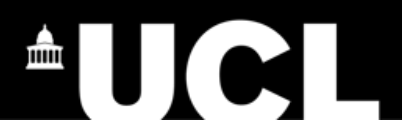

\section{Thinking is Now Deeply Unfashionable}
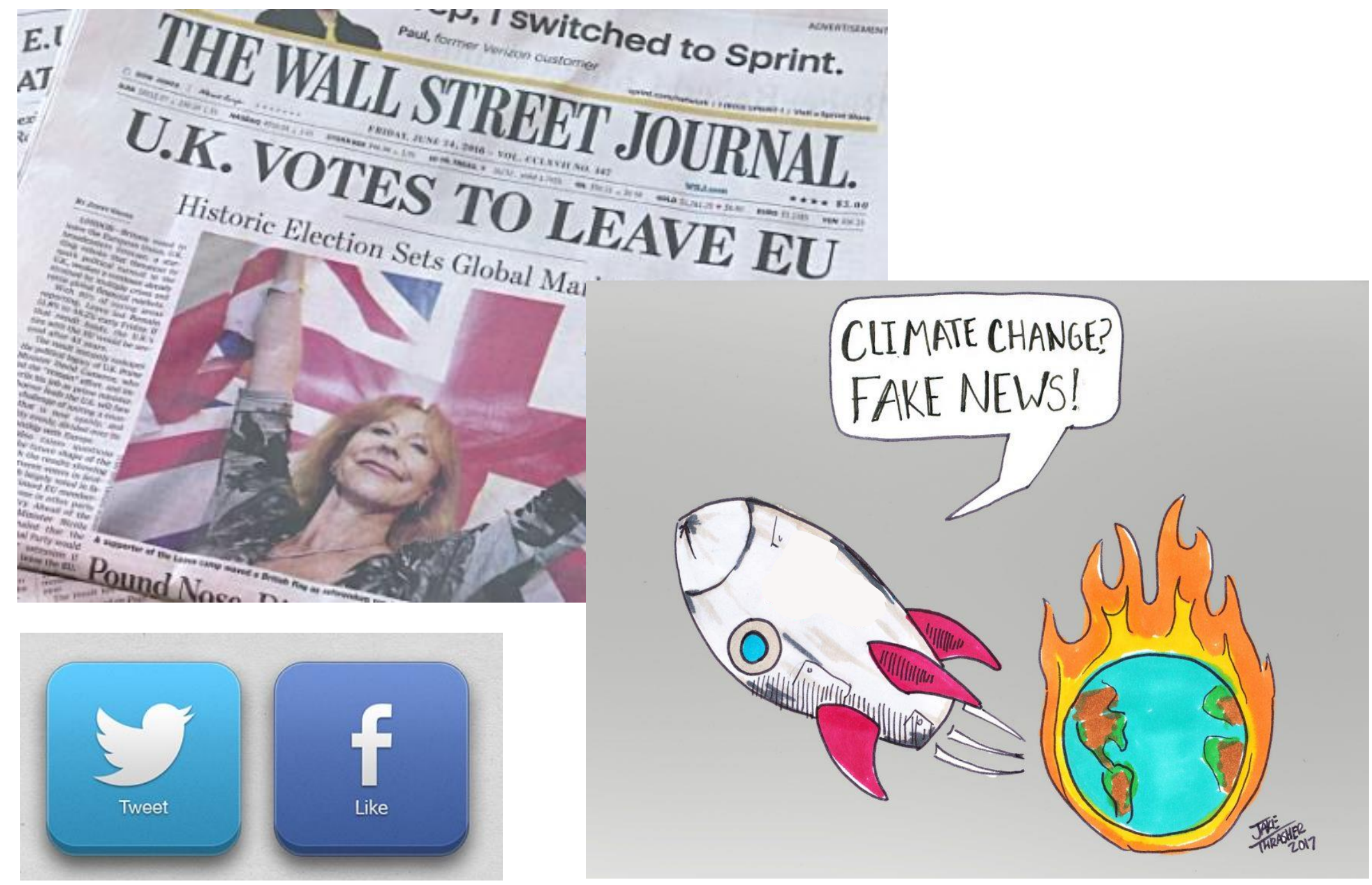


\section{Rational Thought has been made a Disease}

\section{Was Albert Einstein Autistic?}

The boy was an odd one, that was something his family could agree about. When he was born, the back of his head was enormous. His grandmother thought he was just fat, but his parents were worried it was a sign of some problem. But within a few weeks, he'd managed to grow into it somehow, so at least he didn't look strange.

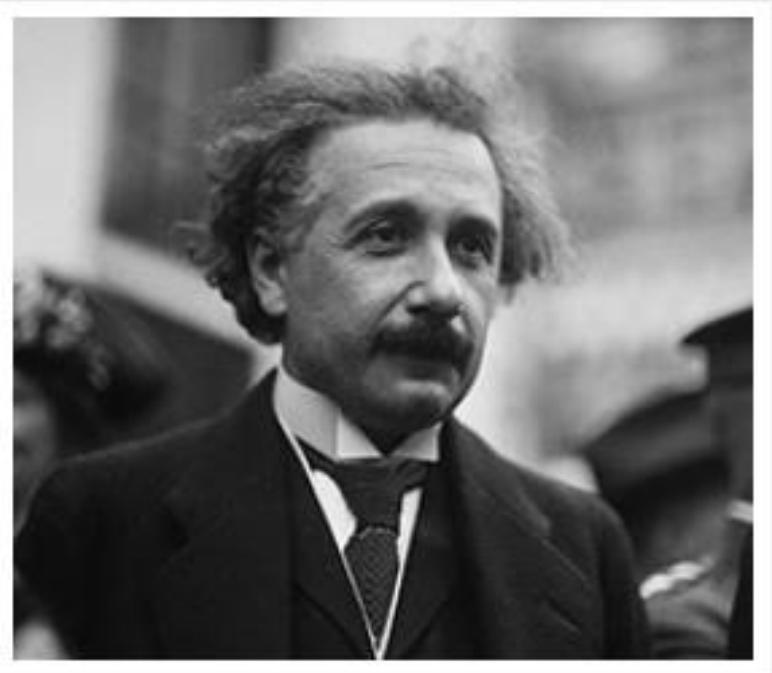

But then, as he grew older, he wouldn't speak! As others his age were learning words and then assembling them into sentences, he 


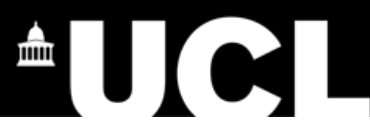

\section{The Artificial Intelligence Approach}

- No thinking required

- No expertise needed

- Even the diagram is simpler

Training

(Test) Data
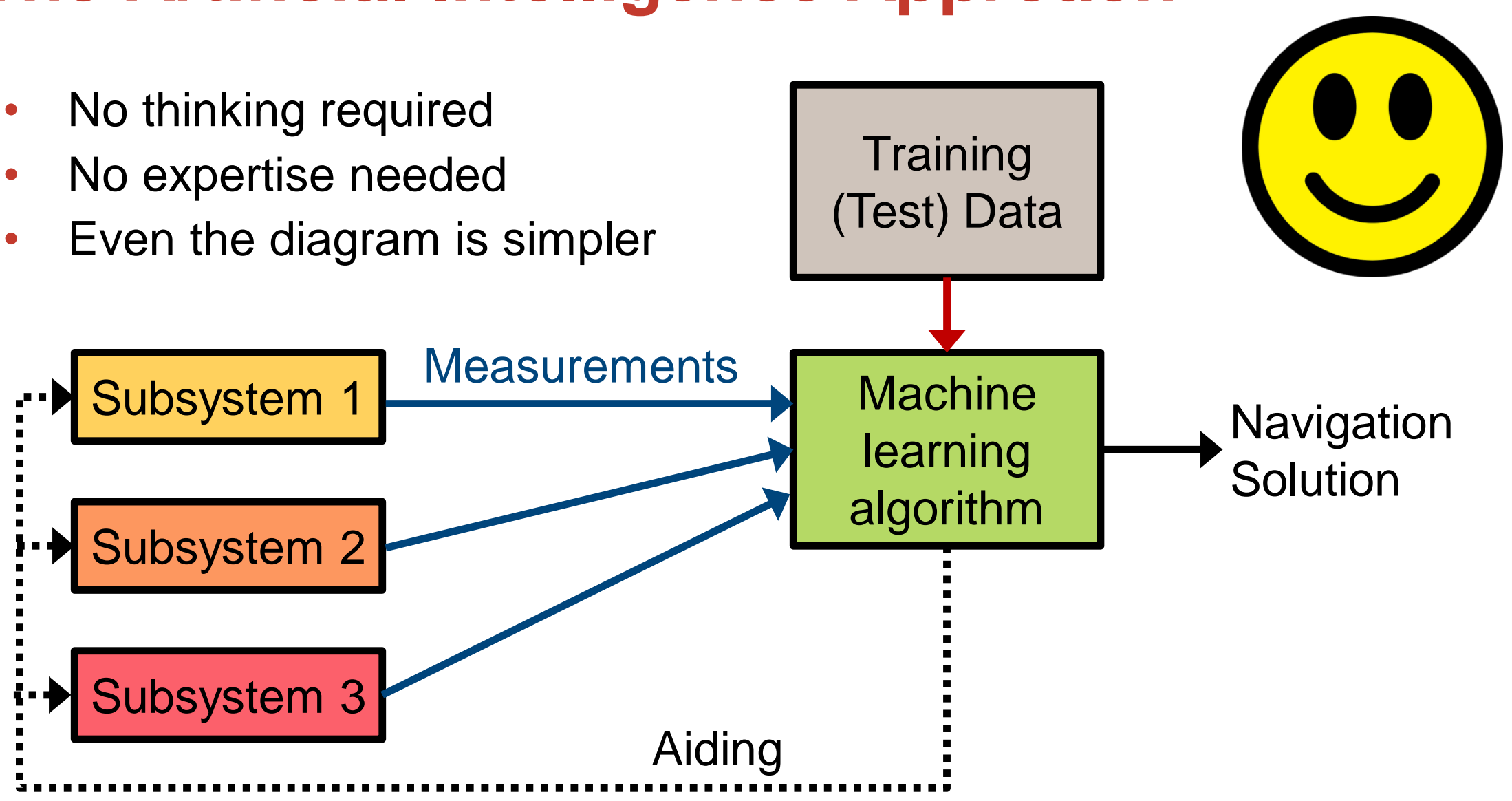

Challenge: Can we trust the navigation solution?

Solution: If you don't employ expert engineers, no-one will ask the question 
Assuming no prior GNSS expertise...

Step 1: Collect training data

- Distribute GNSS receivers across entire area of operation

- Space them at the required positioning resolution

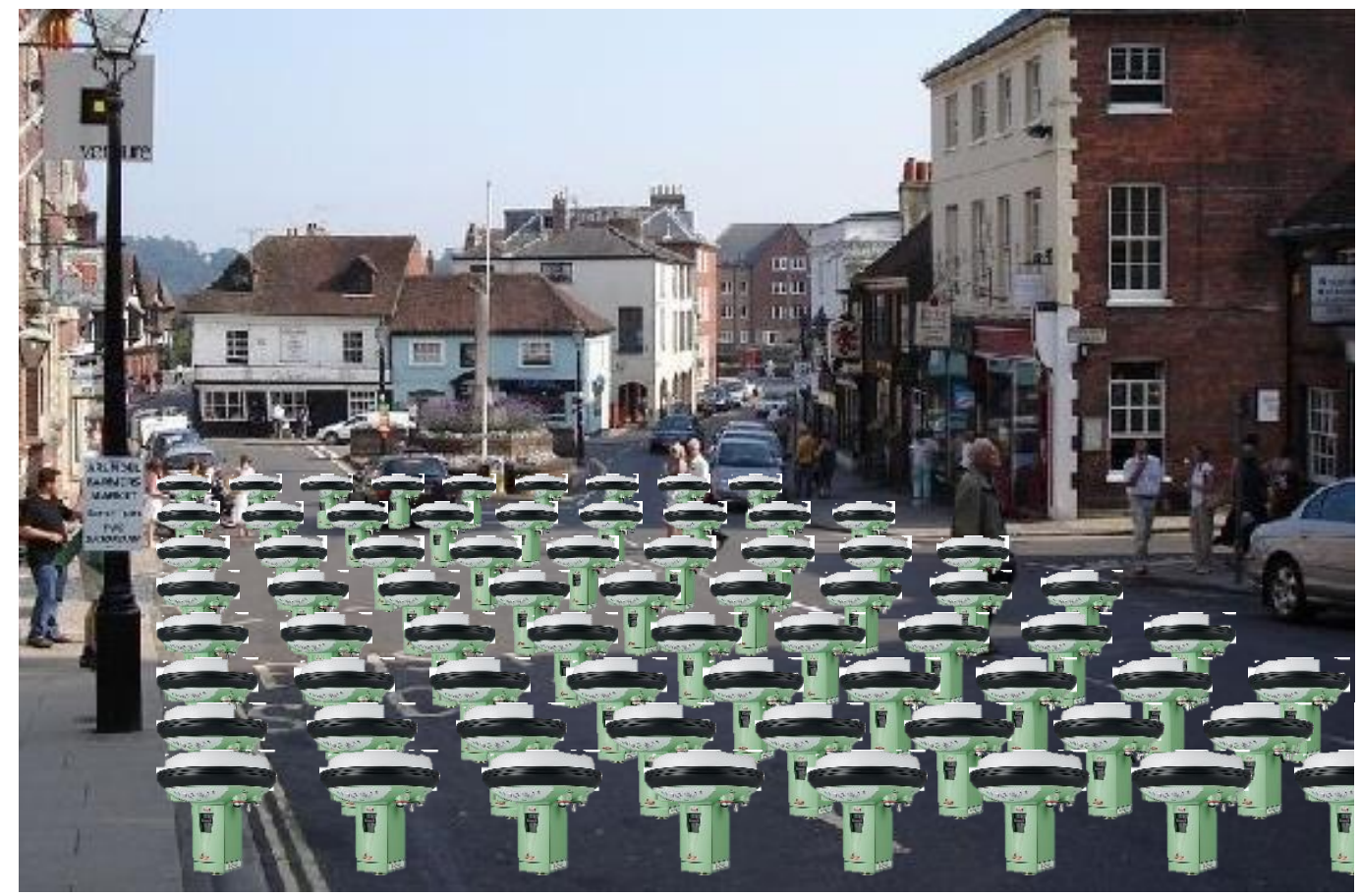


GNSS Positioning Using Machine Learning (2)

Step 2: Train your machine learning algorithm

- Input GNSS receiver ADC outputs and true receiver positions

- $4 \mathrm{MB}$ per second per receiver (2 bit sampling at $16 \mathrm{Msamples/s)}$

- 86,400 s to capture GPS ground track repeat period (longer for other constellations)

- $1 \mathrm{~m}$ resolution

- 350 petabytes of training data per $\mathrm{km}^{2}$ of service area

- This is more data than CERN has

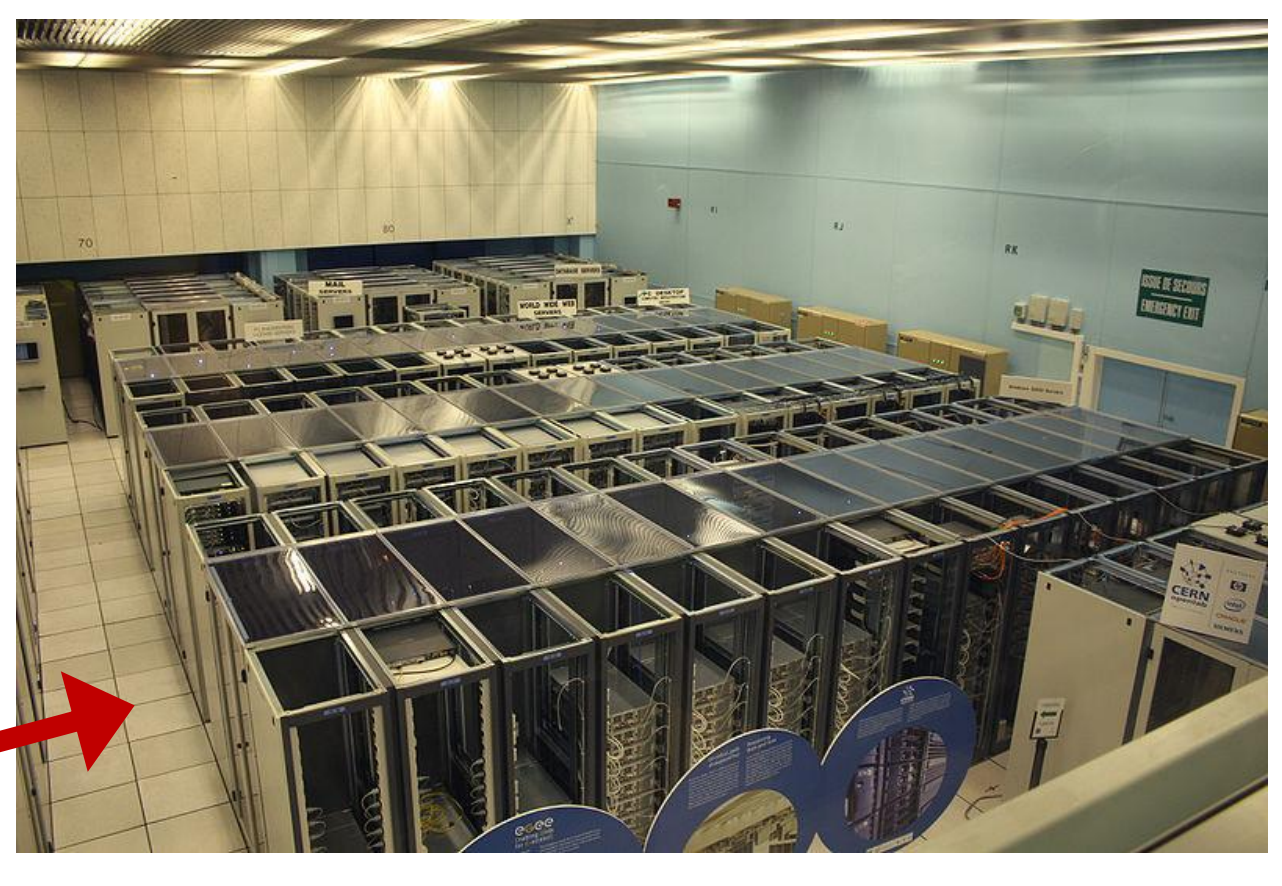


GNSS Positioning Using Machine Learning (3)

Step 3: Positioning service

- Send GNSS receiver ADC outputs (4 kB per ms) to server

- Input to massive deep learning algorithm (too big for a mobile device)

- Return position to user
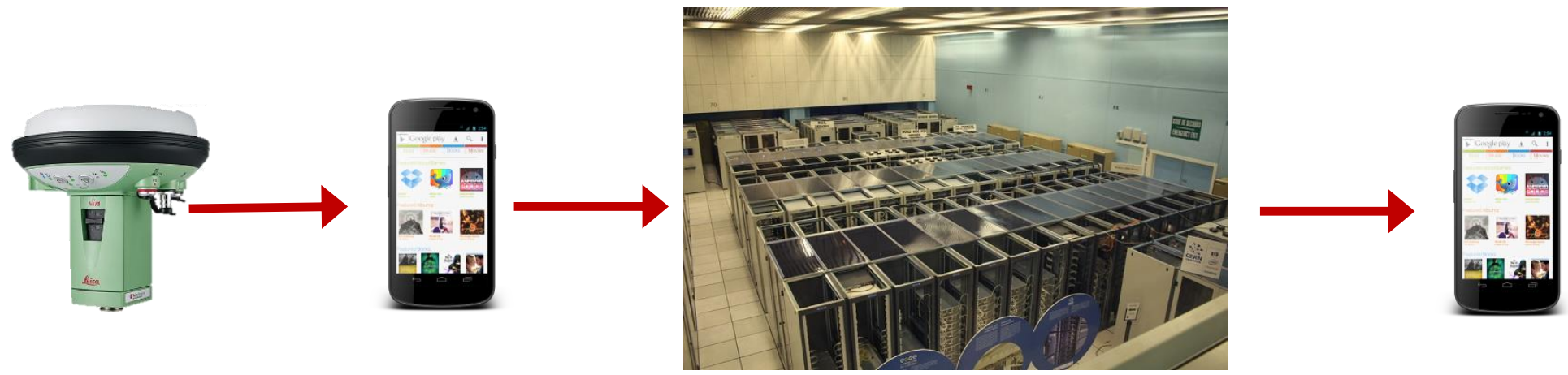

- Correlating the PRN codes, downloading the ephemeris and computing a least-squares solution is much much easier!

Maybe we still need expertise and thinking 


\section{Is Machine Learning Useful at all?}

Physics-based mathematical modelling is more efficient for systems we understand

Machine learning is useful for systems that are difficult to model

- Nonlinear inertial sensor errors

- Object recognition

- Context determination

But... thinking is still needed

- Define classes to recognise or parameters to estimate

- Select the right machine learning algorithm

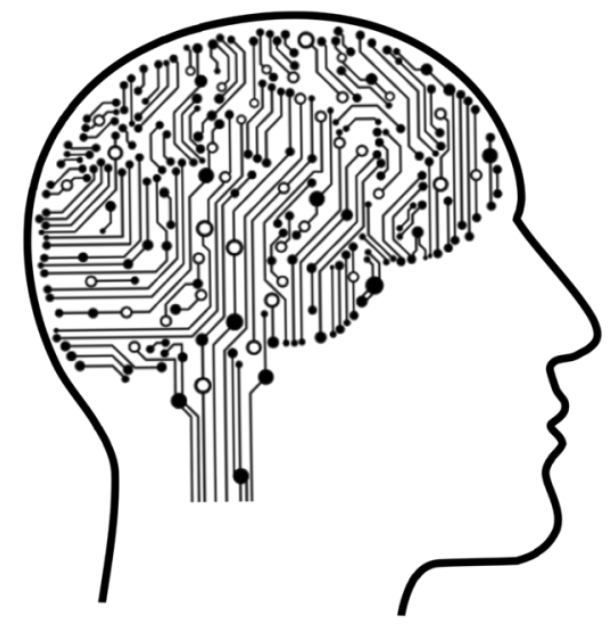

- Determine the feature data to input

- Devise a suitable training process 


\section{Fault Detection (1)}

\section{Multi-sensor fault detection}

- More measurements improves outlier detection sensitivity

- But more measurements also makes simultaneous faults more likely

- And simultaneous faults are not necessarily independent

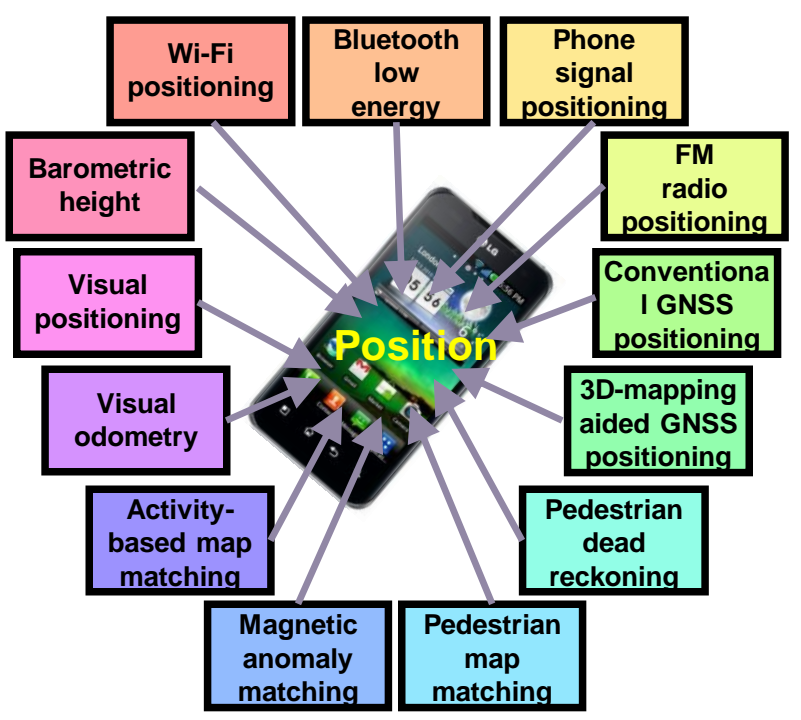

\section{Multi-epoch fault detection}

- More measurements improves outlier detection sensitivity

- But there are more faults to detect

- And faults are often correlated over successive epochs

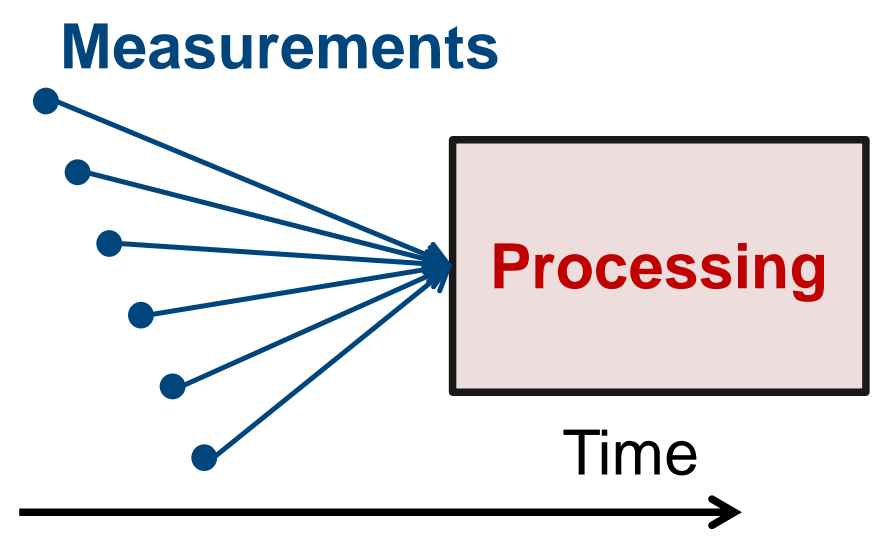




\section{Fault Detection (2)}

\section{Recursive Estimator}

- Limited processing load for unlimited epochs

- Faults must be detected immediately to avoid contaminating the state estimates

\section{Batch Estimator}

- Processing load increases with number of epochs, effectively limiting the number of epochs

- Faulty measurements can be removed at any time

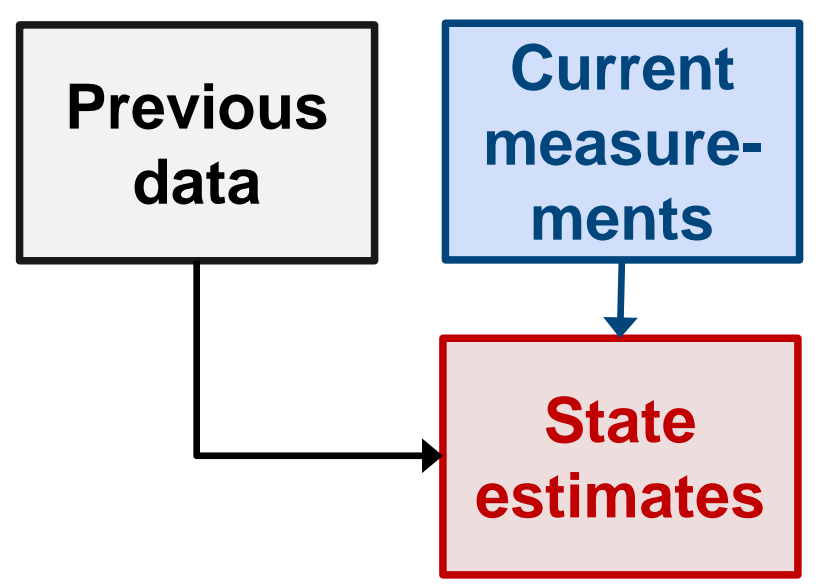

Measurements

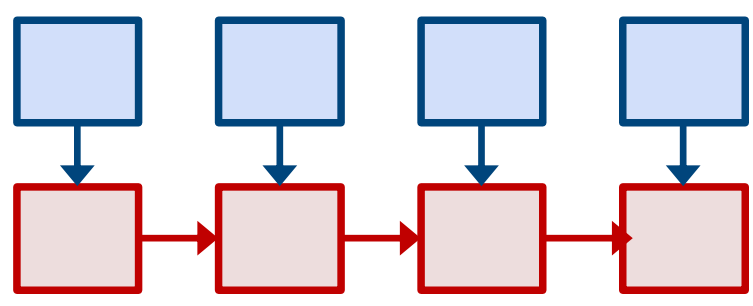

State estimates

Time 


\section{Fault Detection (3)}

\section{Hybrid Batch-Recursive Estimator}

- Most recent epochs considered separately with older epochs combined recursively

- Processing load is finite for unlimited epochs

- Faulty measurements can be removed at any time before they are absorbed in the recursive part of the filter

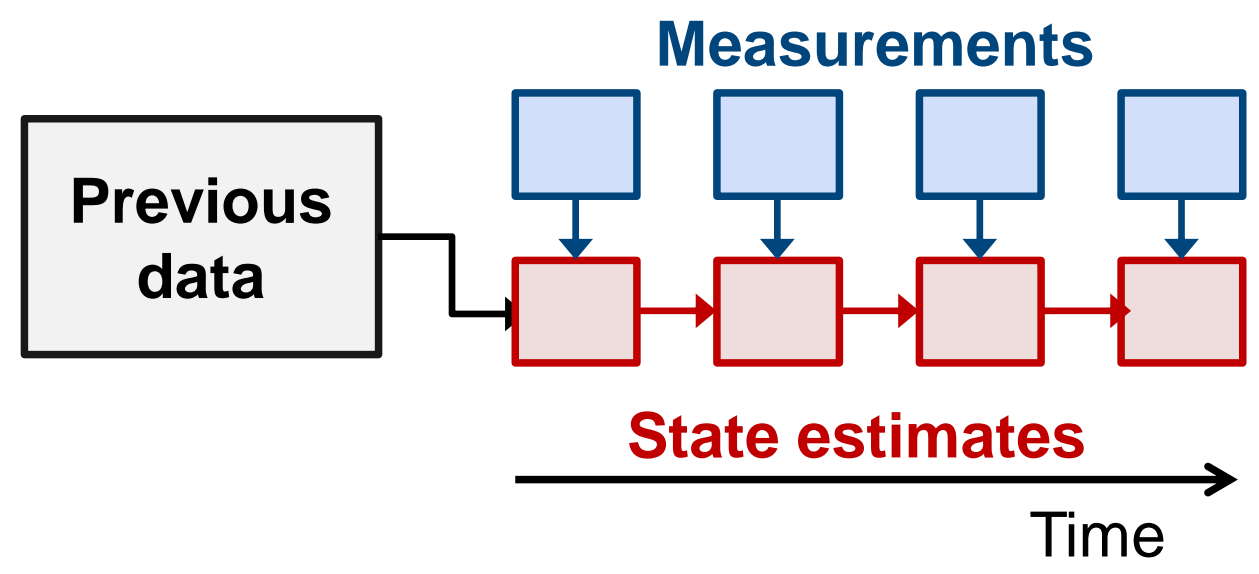

- Fault detection algorithms must consider multiple faults that can be correlated over time and across different measurements 


\section{Some Thoughts on Integrity}

Formal Integrity Requirements:

- The probability that the position error exceeds $x$ must be less than $y$ otherwise an alert must be raised

This requires real-time calculation of

- Either Integrity risk of a position error exceeding $x$

- Or position error corresponding to an integrity risk of $y$

Which requires..

- Statistical error distribution of all measurements under normal operation and for each failure mode

- Probability of each failure mode

- Temporal correlation models for all error sources

- Models of error correlation across measurements for all sources

I don't know how to do this! 


\section{Summary and Discussion Points}

Context determination allows a navigation system to adapt to different environments and user behaviours

Do you agree?

Plug'n'play multisensor integration helps to manage complexity But is it practical?

Machine learning is not a replacement for Bayesian estimation But, does it still have uses in navigation?

A hybrid batch-recursive estimator is a good approach to multiepoch multi-sensor Fault Detection Are there any alternative proposals?

Maintaining integrity in a complex multisensor system is very difficult. Does anyone have any suggestions? 